\title{
NUDT1-Knockdown Inhibits Proliferation, Invasion and Migration of CAL27
}

Qiusheng Shan ( $\nabla$ p0h53x0n@s.okayama-u.ac.jp )

Okayama University Graduate School of Medicine

Research article

Keywords: OSCC, Bioinformatics, Proliferation, Apoptosis, Invasion, Migration

Posted Date: August 26th, 2020

DOl: https://doi.org/10.21203/rs.3.rs-56328/v1

License: (9) This work is licensed under a Creative Commons Attribution 4.0 International License. Read Full License 


\section{Abstract}

Background: The high level of reactive oxygen species (ROS) in cancer could oxidize guanine into 8-oxoG resulting in A: T changed into G: C or G: $\mathrm{C}$ into A: T. Nudix hydrolase 1 (NUDT1) overexpressed in many kind of cancers could hydrolyze the 8-oxo-dGTP into 8-oxo-dGMP or pyrophosphate to prevent the DNA damage or gene mutation. However, the role and function of NUDT1 in oral squamous cell carcinoma (OSCC) has not been investigated.

Methods: Firstly, the bioinformatics methods were used to predict the biological process, cellular component and molecular function of NUDT1 and to confirm the mRNA and protein expression level of NUDT1 in OSCC. Furthermore, the MTT assay, Flow Cytometry, Transwell (Invasion), Scratch Test and Transwell (Migration) were used to test the effect of NUDT1 inhibitor on the proliferation, cell apoptosis, invasion and migration of CAL27. In addition, western blot was used to exam the expression level of NUDT1 and cleaved caspase-3 in different groups. Finally, the laser scanning microscope (LSM) was used to test the invadopodia formation level in different groups.

Results: The bioinformatics analysis suggested that both mRNA and protein of NUDT1 overexpressed in head and neck squamous cell carcinoma (HNSCC) and OSCC. Furthermore, the biological process of NUDT1 mainly enriched in response to oxidative stress, aging, response to cadmium ion and male gonad development in OSCC. the cellular component of NUDT1 mainly enriched in extracellular exosome, cytoplasm, plasma membrane and nucleus in OSCC. The molecular function of NUDT1 mainly enriched in protein binding in OSCC. Finally, the biological experiments confirmed that NUDT1-knockdown could inhibit the proliferation, invasion, migration and promote cell apoptosis of CAL27.

Conclusion: The overexpressed NUDT1 is positively associated with the progression of OSCC which has much potential to be new target in targeted therapy of OSCC.

\section{Background}

OSCC is the most common cancer in head and neck region, which account for $90 \%$ of oral malignancies $[1,2]$. Although improved in diagnosis and treatment methods, the five-year survival of OSCC patients is still high about $60-65 \%$, which has negative effect on the life quality of patients [3,4]. The targeted therapy included oncogene targeted therapy and non-oncogene targeted therapy could targeted on specific genes to inhibit the progression of OSCC with little side effect $[5,6]$. Therefore, it is quite necessary to study the biomarkers associated with the progression of OSCC to improve the treatment effect and life quality of OSCC patients.

The high level of ROS in cancer could induce many different types of biological process such as proliferation, DNA damage and cell death [7]. Due to the low redox potential, the guanine could be oxidized into 8-oxoG by ROS more easily resulting in A: T changed into G: C or G: C into A: T, which may cause DNA damage and gene mutation [8]. NUDT1 plays a significant role in the progression of many kind of cancers, which could hydrolyze the 8-oxo-dGTP into 8-oxo-dGMP or pyrophosphate to prevent the 
DNA damage or gene mutation [9-12]. Therefore, NUDT1 could prevent the apoptosis caused by gene mutation and maintain the progression of cancer. Recent study indicated that the NUDT1-knockdown could inhibit the proliferation of gastric cancer cells and lung cancer cells $[9,10]$. In addition, NUDT1knockdown could also inhibit the invasion and metastasis of gastric cancer and hepatocellular carcinoma $[9,13]$. Therefore, NUDT1 is quite associated with the progression of many kind of cancers but study little in OSCC.

we hypothesis that NUDT1 is quite associated with the progression of OSCC, which can be a new biomarker in targeted therapy of OSCC. To valid this hypothesis, the bioinformatics methods were firstly used to predict the mRNA and protein expression level, biological process, cell component and molecular function of NUDT1. Furthermore, the biological experiments were conducted to test the role and function of NUDT1 in the progression of OSCC.

\section{Methods}

\section{Datasets}

The gene expression profiles of GSE13601 and GSE138206 were obtained from The Gene Expression Omnibus (GEO, http://www.ncbi.nlm.nih.gov/geo). The data of GSE13601 was submitted by Singh B, Estillo $C$ and Socci ND, which includes 37 OSCC tissues and 20 normal tissues. The data of GSE138206 was submitted by Pan $\mathrm{H}$, Wang $\mathrm{Y}$ and Tang Z, which includes 6 OSCC tissues and 6 normal tissues. The transcript data of HNSCC were downloaded from The Cancer Genome Atlas (TCGA) included 501 HNSCC tissues and 43 normal tissues.

\section{Data processing}

The data of GSE13601 and GSE138206 were normalized to identified the differentially expressed genes (DEGs) by R 3.6.2 (limma package), which results presented by Volcano plot. The common DEGs between GSE13601 and GSE138206 was presented by Venn plot, which was made by Venny2.1.0. (https://bioinfogp.cnb.csic.es/tools/venny/index.html). $P<0.05$ and |LogFC| $>1$ was defined as cutoff value to identify DEGs.

\section{GO enrichment analysis and KEGG enrichment analysis.}

The biological process, cell component, molecular function and cell pathways of common DEGs were analyzed by Gene Ontology (GO) and Kyoto Encyclopedia of Genes and Genomes (KEGG) enrichment analysis through DAVID and the results were presented by bubble plot made by $\mathrm{R}$ 3.6.2. $P<0.05$ was considered as cutoff value. 


\section{Verification the mRNA and protein expression level of NUDT1 by TCGA dataset and The Human Protein Atlas.}

The transcript data in TCGA dataset was downloaded and normalized by Log2. The NUDT1 mRNA expression level was presented by violin plot made by graphpad prism 8.0.1. In addition, The NUDT1 mRNA expression level in GSE13601 and GSE138206 was presented by violin plot after normalized by Log2. The NUDT1 protein expression level was confirmed by The Human Protein Atlas. The expression of NUDT1 in oral mucosa tissues was regarded as control group and the expression of NUDT1 in HNSCC tissues was regarded as experimental group. The compare of expression level concerned both intensity and the percentage of positive cells.

\section{Cell culture}

TH287 provided by Dalian University of Technology was used as NUDT1 inhibitor. The human oral cancer cell line CAL27 was purchased from American Type Culture Collection (ATCC, Manassas, VA, USA). Cells were cultured in high-glucose DMEM (GE Healthcare HyClone, Logan, UT, USA) supplemented with $10 \%$ fetal bovine serum (BI, Kibbutz Beit Haemek, Israel) and 1\% antibiotics (Beyotime, Biotechnology, Jiangsu, China). All cells were grown in a humidified atmosphere of $5 \% \mathrm{CO}_{2}$ and $95 \%$ air at $37^{\circ} \mathrm{C}$.

\section{MTT assay}

The cells were seeded in the 96 -well plate with the density of $5 \times 10^{3} /$ well. After $12 \mathrm{~h}$, the increasing dose of NUDT1 inhibitor $(0.1,1,10,100,1000 \mu \mathrm{M})$ was added into the plate. After incubating for $72 \mathrm{~h}, 20 \mu \mathrm{L}$ MTT reagent $(0.5 \mathrm{mg} / \mathrm{mL})$ was added into each well and incubate for another $4 \mathrm{~h}$ at $37^{\circ} \mathrm{C}$. Finally, Supernatants were subsequently discarded and $100 \mu \mathrm{L}$ DMSO was added into each well. The absorbance was measured by enzyme-linked immunosorbent assay reader at $490 \mathrm{~nm}$. The data analysis was conducted by the graphpad prsim 8.0.1.

\section{Flow cytometry}

The cells in different groups were centrifuged $(1000 \mathrm{r} / \mathrm{min}, 5 \mathrm{~min})$ after digested by trypsin without EDTA and resuspended by precooled PBS. $5 \times 10^{5}$ cells were added into each flow cytometry tube after washing by precooled PBS for three times. Resuspend the cells by $200 \mu \mathrm{L}$ buffer liquids and add Annexin $\mathrm{V}$ and FITC/PI. After incubating for $15 \mathrm{~min}$ in room temperature, another $100 \mu \mathrm{L}$ buffer liquid was added into each tube which was filtered before tested by Flow cytometry machine (FACS-can, BD bioscience).

\section{Western Blot}


Cells were collected and proteins were extracted by RIPA buffer (Beyotime, Jiang Su, China) containing 1x Protease Inhibitor Cocktail. Protein concentration was tested by BCA Protein Assay Kit (Beyotime, Jiang $\mathrm{Su}, \mathrm{China}$ ). Total proteins were separated by $12.5 \%$ sodium dodecyl sulfate polyacrylamide gel electrophoresis transferred onto Nitrocellulose membrane (Millipore, MA, USA) and blocked in blocking solution on a shaker for $2 \mathrm{~h}$ at room temperature. And then, incubate it with primary antibodies including $\beta$-actin (1:1000, CST), NUDT1 (1:2000, Abcam) and cleaved caspase-3 (1:1000, CST) diluted in blocking solution ( $5 \%$ non-fat milk dissolved in Tris-buffered saline (TBS)-Tween-20) overnight at $4{ }^{\circ} \mathrm{C}$ respectively. After washing by TBST for $30 \mathrm{~min}$, the membranes were incubated with flourophore-conjugated second antibody (1:8000, CST) for $2 \mathrm{~h}$ and wash it for six times in 30 min with $15 \mathrm{~mL}$ of TBST. Finally, scanning membrane by fluorescent scanner after draining membrane of excess TBST.

\section{Transwell (Invasion)}

The $50 \mu \mathrm{L}$ mixed solution made by Matrigel and DMEM according to 1:5 was added into bottom of transwell chamber which was incubated for $2 \mathrm{~h}$ in the incubator with $5 \% \mathrm{CO}^{2}$ and $95 \%$ air at $37^{\circ} \mathrm{C}$. After discarding the mixed solution, another $50 \mu \mathrm{L}$ DMEM was added into the chamber for hydration $30 \mathrm{~min}$. The cells were treated by NUDT1 inhibitor and DMEM for $24 \mathrm{~h}$ respectively. After that, the cells were digested and centrifuged normally and resuspended by DMEM. Adjust the cell density to $1 \times 10^{5} / 200 \mu \mathrm{L}$ and add $200 \mu \mathrm{L}$ cell suspension into up chamber. Another $500 \mu \mathrm{L}$ DMEM with $10 \%$ FBS was added into low chamber. After incubating for $48 \mathrm{~h}$ in $5 \% \mathrm{CO}^{2}$ and $95 \%$ air at $37{ }^{\circ} \mathrm{C}$, the cells in up chamber were removed by cotton swab slightly and washed by PBS for $5 \mathrm{~min}$. After fixed by methanol for $15 \mathrm{~min}$, the chambers were stained by $0.1 \%$ crystal violet staining solution for $30 \mathrm{~min}$ in dark. Finally, taking pictures by $200 \times$ inverted microscope after washing by PBS for three times. The data was analyzed by Image J.

\section{Laser Scanning Microscope}

Cells were added into LSM specific dish at a density of $2 \times 10^{5} /$ well and incubated for $24 \mathrm{~h}$. After that, the DMEM and NUDT1 inhibitor were added into control group and experimental group respectively. After incubating for $24 \mathrm{~h}$, the Hoechst $(50 \mu \mathrm{L})$ was added into each dish to incubate for 20 min before sample collecting. Wash each dish with PBS for three times followed by fixed the cells with $4 \%$ paraformaldehyde for 30 min after discarding the medium. Sealed the sample with 5\% BSA (dissolved in PBS) for $2 \mathrm{~h}$ after washing it with PBS for three times. The Cortactin antibody (CST, 1:200 diluted in 1\% BSA) was added into the dishes which were incubated at $4^{\circ} \mathrm{C}$ overnight. Finally, the Alex594 (1:200, diluted in 1\%BSA) was added into the dishes, which were incubated for $2 \mathrm{~h}$ at $37^{\circ} \mathrm{C}$ in dark. Taking pictures by $400 \times$ inverted laser scanning microscope after washing by PBS for three times. The data analysis was conducted by Image Pro Plus 6.0.

\section{Transwell (Migration)}


The cells were treated by NUDT1 inhibitor and DMEM for $24 \mathrm{~h}$ respectively. After that the cells were digested and centrifuged normally and resuspended by DMEM. Adjust the cell density to $2 \times 10^{4} / 200 \mu \mathrm{L}$ and add $200 \mu \mathrm{L}$ cell suspension into up chamber. Another $500 \mu \mathrm{L}$ DMEM with $10 \%$ FBS was added into low chamber. After incubating for $48 \mathrm{~h}$ in $5 \% \mathrm{CO}^{2}$ and $95 \%$ air at $37{ }^{\circ} \mathrm{C}$, the cells in up chamber were removed by cotton swab slightly and washed by PBS for 5 min. After fixed by methanol for $15 \mathrm{~min}$, the chambers were stained by $0.1 \%$ crystal violet staining solution for $30 \mathrm{~min}$ in dark. Finally, taking pictures by $200 \times$ inverted microscope after washing by PBS for three times. The data was analyzed by Image J.

\section{Scratch Test}

The cells were digested and centrifuged normally after approaching $90 \%$ density and adjusted the cells density at $1 \times 10^{6} / \mathrm{mL} .1 \mathrm{~mL}$ medium and $1 \mathrm{~mL}$ cell suspension were added into each well in 6-well plate, which was incubated in the incubator with $5 \% \mathrm{CO}^{2}$ and $95 \%$ air at $37^{\circ} \mathrm{C}$. After the cells covered the well, scratch the well for three straight lines by $20 \mu \mathrm{L}$ pipette tips. After discarding the liquid and washing by PBS, add the DMEM in control group and NUDT1 inhibitor $(100 \mu \mathrm{M})$ in experimental group. Finally, taking pictures by 200x inverted microscope at $0 \mathrm{~h}, 6 \mathrm{~h}, 12 \mathrm{~h}$ and $24 \mathrm{~h}$ respectively. The data was analyzed by Image Pro Plus 6.0.

\section{Statistics Analysis}

The data was analyzed by graphpad prism 8.0.1 (San Diego, CA, USA). All of data are shown as mean \pm s.d. (s.d., standard deviation). The comparison of data between two varied factors were conducted by two-way ANOVA. The comparison of data between two groups were analyzed by the student's t-test. Values of at least $P<0.05$ were considered statistically significance.

\section{Results}

\section{Identification of common DEGs between GSE13601 and GSE138206.}

The data of GSE13601 and GSE138206 were normalized to identify the DEGs by R3.6.2 (Fig.1 A, B), which indicated that there are 4256 and 1959 DEGs in GSE13601 and GSE138206 respectively (Fig.1 C, D). The common DEGs between GSE13601 and GSE138206 were identified by Venn, which demonstrated that there are 671 common DEGs between GSE13601 and GSE138206 (Fig.1 E). All of these data indicated that there are 671 common DEGs in OSCC.

\section{GO and KEGG enrichment analysis.}


The biological process, cell component and molecular function of these 671 common DEGs were identified by GO enrichment analysis (Fig.2 A, B, C). In addition, the cell pathways of these 671 common DEGs were analyzed by KEGG enrichment analysis (Fig.2 D).

\section{The biological process, cell component and molecular function of NUDT1 was identified by GO enrichment analysis.}

The biological process of NUDT1 was presented by bubble plot which indicated that the NUDT1 mainly enriched in response to oxidative stress, aging, response to cadmium ion and male gonad development in OSCC (Fig.3 A). The cell component of NUDT1 was also presented by bubble plot, which demonstrated that the NUDT1 mainly enriched in extracellular exosome, cytoplasm, plasma membrane and nucleus in OSCC (Fig.3 B). Finally, the molecular function of NUDT1 mainly enriched in protein binding (Fig.3 C).

\section{The relative mRNA and protein expression level of NUDT1 were confirmed by TCGA dataset and The Human Protein Atlas respectively.}

The mRNA expression data of NUDT1 was confirmed by TCGA dataset, GSE13601 and GSE138206, which suggested that the NUDT1 up-regulated in HNSCC tissues (Fig.4 A) and OSCC tissues (Fig.4 B, C). In addition, the protein expression data of NUDT1 was downloaded from The Human Protein Atlas, which demonstrated that the NUDT1 up-regulated in HNSCC tissues compared with normal oral mucosa tissues (Fig.4 D). All of these data indicated that both the mRNA and protein of NUDT1 overexpressed in HNSCC tissues and OSCC tissues.

\section{NUDT1-knockdown could inhibit the proliferation of CAL27 by promoting cell apoptosis.}

The effect of NUDT1 inhibitor on the proliferation of CAL27 was measured by MTT assay, which indicated that the NUDT1-knockdown could inhibit the proliferation of CAL27 and the appropriate concentration of NUDT1 inhibitor was nearly $100 \mu \mathrm{M}$ (Fig.5 A). In addition, the effect of NUDT1 inhibitor on the apoptosis of CAL27 was tested by flow cytometry (Fig.5 B), which suggested the NUDT1knockdown could promote the apoptosis of CAL27 (Fig.5 C). Finally, the western blot was used to test the relative NUDT1 and cleaved caspase-3 protein expression level (Fig. 5 D), which demonstrated that the NUDT1 inhibitor could inhibit the expression level of NUDT1 effectively leading to the increase in the expression level of cleaved caspase-3 (Fig. 5 E, F). All of these data suggested that the NUDT1 inhibitor could knockdown the NUDT1 effectively resulting in the promotion of cell apoptosis of CAL27. 


\section{NUDT1-knockdown could inhibit the invasion of CAL27 by suppressing invadopodia formation.}

The effect of NUDT1 inhibitor on the invasion of CAL27 was examined by Transwell (Invasion) (Fig.6 A), which indicated that NUDT1-knockdown could inhibit the invasion of CAL27 (Fig.6 B). In addition, the effect of NUDT1 inhibitor on the invadopodia formation of CAL27 was measured by Laser Scanning Microscope (Fig.6 C), which suggested that both the cells with invadopodia and the number of invadopodia per cell decreased dramatically after knockdown of NUDT1 (Fig.6 D, E). All of these data demonstrated that NUDT1-knockdown could inhibit the invasion of CAL27 by suppressing invadopodia formation.

\section{NUDT1-knockdown could inhibit the migration of CAL27.}

The effect of NUDT1 inhibitor on the migration of CAL27 was tested by both Transwell (Migration) (Fig.7 A) and Scratch Test (Fig.7 C), which results suggested that both the migration cell number (Fig.7 B) and migration distance (Fig.7 D) of CAL27 decreased after knockdown of NUDT1. All of these results indicated that NUDT1-knockdown could inhibit the migration of CAL27.

\section{Discussion}

The bioinformatics analysis indicated that both mRNA and protein of NUDT1 overexpressed in HNSCC and OSCC. Furthermore, the biological process of NUDT1 mainly enriched in response to oxidative stress, aging, response to cadmium ion and male gonad development in OSCC. the cellular component of NUDT1 mainly enriched in extracellular exosome, cytoplasm, plasma membrane and nucleus in OSCC. The molecular function of NUDT1 mainly enriched in protein binding. Finally, the biological experiment confirmed that NUDT1-knockdown could inhibit the proliferation, invasion, migration and promote cell apoptosis of CAL27.

Compared with normal cells, there is high level of ROS in cancer cell associated with cellular transformation, inflammation, tumor survival, proliferation, invasion, migration and even epigenetic gene change [14]. DNA damage caused by ROS includes DNA base lesions, DNA sugar lesion and DNA strand break. Furthermore, the guanine that has low oxidation potential could be oxidized more easily compared with the other nucleotides $[8,15]$. The oxidized form of guanine is 8-oxoG, which could incorporate into the DNA during DNA replication and strand breaking leading to the transversion mutation that G: C into A: T or A: T into G: C $[16,17]$. NUDT1 plays a significant role in carcinogenesis [18]. The expression level of NUDT1 is higher than the adjacent normal tissues in the oncogenic KRAS-driven cancers such as pancreatic and lung cancer [19]. The main protein production of NUDT1 is MTH1 protein, which expressed in cytoplasm and could hydrolyze the 8-oxo-dGTP into 8-oxo-dGMP or pyrophosphate [12, 20]. Therefore, the gene mutation caused by 8-oxo-dGTP was inhibited by overexpressed NUDT1 and the cancer cell apoptosis could not be activated [21]. Recent study indicated that the NUDT is a potential 
gene associated with germline mutation of non-small cell lung cancer [22]. Another research also found that the NUDT1 is quite associated with the progression of non-small cell lung cancer [23]. In this study, we also found that both the mRNA and protein of NUDT1 overexpressed in OSCC tissues. In addition, the main function of NUDT1 is oxidative stress, aging, response to cadmium ion and male gonad development in OSCC. And the NUDT1 mainly expressed in extracellular exosome, cytoplasm, plasma membrane and nucleus in OSCC. all of these findings are similar to the above-mentioned research work in non-small cell lung cancer, which means that the NUDT1 is also a potential gene associated with the progression of OSCC.

The overexpressed NUDT1 is quite associated with the proliferation of many kind of cancers. Recent study demonstrated that NUDT1-knockdown could inhibit the proliferation of gastric cancer and lung cancer $[9,10,24]$. We found that NUDT1-knockdown could also inhibit the proliferation of CAL27, which also supported the function of overexpressed NUDT1 in gastric cancer and lung cancer in abovementioned research works indirectly. In addition, some researchers also found that the overexpressed NUDT1 is quite associated with invasion of many kind of cancers. NUDT1-knockdown also inhibits the invasion of gastric cancer and hepatocellular carcinoma [9, 13], while has not been investigated in OSCC. The invasion ability of OSCC was regulated by invadopodia formation level [25]. During the invasion movement of OSCC, the cancer cells will generate the invadopodia firstly, which could secret the MMP2, MMP9 and MMP14 to break up the local and distance extracellular matrix respectively $[26,27]$.

Furthermore, the invadopodia formation of OSCC was regulated by the Epidermal Growth Factor Receptor (EGFR) signal [28]. In this study, we found that the NUDT1-knockdown could inhibit the invasion of CAL27 by suppressing the invadopodia formation. However, whether NUDT1 could regulate the invadopodia formation of CAL27 by EGFR signal need to be further studied. The knockdown of NUDT1 could inhibit the migration of osteosarcoma cells [29]. And the knockdown of NUDT1 could also inhibit the migration of gastric cancer through PI3K/AKT pathway [30]. Therefore, overexpressed NUDT1 is quite associated with the migration of many kind of cancers. Epithelial-to-mesenchymal transition (EMT) process plays a significant role in the migration of OSCC [31]. Recent study demonstrated that the hypoxia could induce the EMT by PI3K/AKT pathway, which could promote the metastasis of OSCC [32]. In addition, EMT process was also regulated by wnt10b cell pathway in OSCC [33]. In this study, we found that NUDT1knockdown could also inhibit the migration of CAL27. However, whether NUDT1 could regulate the migration of CAL27 by EMT process need to be further studied.

\section{Conclusion}

NUDT1-knockdown could inhibit the proliferation and promote the activation of cell apoptosis by preventing gene mutation in CAL27. In addition, NUDT1-knockdown could also inhibit invasion and migration of CAL27. All of these results suggested that the overexpressed NUDT1 is quite associated with the progression of OSCC, which has much potential to be new biomarkers in targeted therapy of OSCC.

\section{Abbreviations}


ROS: Reactive Oxygen Species

NUDT1: Nudix hydrolase 1

OSCC: Oral Squamous Cell Carcinoma

HNSCC: Head and Neck Squamous Cell Carcinoma

LSM: Laser Scanning Microscope

TCGA: The Cancer Genome Atlas

GEO: Gene Expression Omnibus

DEGs: Differentially Expressed Genes

GO: Gene Ontology

KEGG: Kyoto Encyclopedia of Genes and Genomes

EGFR: Epidermal Growth Factor Receptor

EMT: Epithelial-to-Mesenchymal Transition

\section{Declarations}

\section{Ethics approval and consent to participate}

Not applicable

\section{Consent for publication}

Not applicable

\section{Availability of data and material}

All data generated or analyzed during this study are included in this published article.

\section{Competing interests}

The authors declare no conflict of interests. 


\section{Funding}

Funding information is not applicable.

\section{Author contributions}

QS designed and conducted the experiment.

\section{Acknowledgements}

Not applicable

\section{References}

1. Li M, Gao F, Yu X, et al. Promotion of ubiquitination-dependent survivin destruction contributes to xanthohumol-mediated tumor suppression and overcomes radioresistance in human oral squamous cell carcinoma. J Exp Clin Cancer Res. 2020;39(1):88.

2. Lindemann A, Takahashi $H$, Patel AA, Osman AA, Myers JN. Targeting the DNA damage response in OSCC with TP53 mutations. J Dent Res. 2018;97(6):635-44.

3. Yamamoto T, Hirosue A, Nakamoto M, et al. BRD4 promotes metastatic potential in oral squamous cell carcinoma through the epigenetic regulation of the MMP2 gene. Br J Cancer. 2020.

4. Yang Y, Chen D, Liu H, Yang K. Increased expression of IncRNA CASC9 promotes tumor progression by suppressing autophagy-mediated cell apoptosis via the AKT/mTOR pathway in oral squamous cell carcinoma. Cell Death Dis. 2019;10(2):41.

5. Shakib PA, Ensani F, Abdirad A, Valizadeh B, Seyedmajidi M, Sum S. CD44 and CD74: The promising candidates for molecular targeted therapy in oral squamous cell carcinoma. Dent Res J (Isfahan). 2015;12(2):181-6.

6. Principles of cancer. therapy: oncogene and non-oncogene addiction. Cell. 2009;136(5):823-37.

7. Moloney JN, Cotter TG. ROS signalling in the biology of cancer. Semin Cell Dev Biol. 2018;80:50-64.

8. Boiteux S, Coste F, Castaing B. Repair of 8-oxo-7,8-dihydroguanine in prokaryotic and eukaryotic cells: Properties and biological roles of the Fpg and OGG1 DNA N-glycosylases. Free Radic Biol Med. 2017;107:179-201.

9. Duan J, Zhang H, Li S, et al. The role of miR-485-5p/NUDT1 axis in gastric cancer. Cancer Cell Int. 2017;17:92.

10. Cho WC, Chow AS, Au JS. MiR-145 inhibits cell proliferation of human lung adenocarcinoma by targeting EGFR and NUDT1. RNA Biol. 2011;8(1):125-31.

11. Garre P, Briceño V, Xicola RM, et al. Analysis of the oxidative damage repair genes NUDT1, OGG1, and MUTYH in patients from mismatch repair proficient HNPCC families (MSS-HNPCC). Clin Cancer Res. 
2011;17(7):1701-12.

12. Nakabeppu Y. Cellular levels of 8-oxoguanine in either DNA or the nucleotide pool play pivotal roles in carcinogenesis and survival of cancer cells. Int J Mol Sci. 2014;15(7):12543-57.

13. Ou Q, Ma N, Yu Z, et al. Nudix hydrolase 1 is a prognostic biomarker in hepatocellular carcinoma. Aging. 2020;12(8):7363-79.

14. Prasad S, Gupta SC, Tyagi AK. Reactive oxygen species (ROS) and cancer: Role of antioxidative nutraceuticals. Cancer Lett. 2017;387:95-105.

15. German P, Szaniszlo P, Hajas G, et al. Activation of cellular signaling by 8-oxoguanine DNA glycosylase-1-initiated DNA base excision repair. DNA Repair. 2013;12(10):856-63.

16. Whitaker AM, Schaich MA, Smith MR, Flynn TS, Freudenthal BD. Base excision repair of oxidative DNA damage: from mechanism to disease. Front Biosci (Landmark Ed). 2017;22:1493-522.

17. Scott TL, Rangaswamy S, Wicker CA, Izumi T. Repair of oxidative DNA damage and cancer: recent progress in DNA base excision repair. Antioxid Redox Signal. 2014;20(4):708-26.

18. Bialkowski K, Kasprzak KS. A profile of 8-oxo-dGTPase activities in the $\mathrm{NCl}-60$ human cancer panel: Meta-analytic insight into the regulation and role of MTH1 (NUDT1) gene expression in carcinogenesis. Free Radic Biol Med. 2020;148:1-21.

19. Samaranayake GJ, Huynh M, Rai P. MTH1 as a chemotherapeutic target: the elephant in the room. Cancers (Basel). 2017;9(5):47.

20. Rai P, Sobol RW. Mechanisms of MTH1 inhibition-induced DNA strand breaks: The slippery slope from the oxidized nucleotide pool to genotoxic damage. DNA Repair. 2019;77:18-26.

21. Mur P, Jemth AS, Bevc L, et al. Germline variation in the oxidative DNA repair genes NUDT1 and OGG1 is not associated with hereditary colorectal cancer or polyposis. Hum Mutat. 2018;39(9):1214-25.

22. Luo $W$, Tian $P$, Wang $Y$, et al. Characteristics of genomic alterations of lung adenocarcinoma in young never-smokers. Int J Cancer. 2018;143(7):1696-705.

23. Campbell JM, Lockwood WW, Buys TP, et al. Integrative genomic and gene expression analysis of chromosome 7 identified novel oncogene loci in non-small cell lung cancer. Genome. 2008;51(12):1032-9.

24. Abbas HHK, Alhamoudi KMH, Evans MD, Jones GDD, Foster SS. MTH1 deficiency selectively increases non-cytotoxic oxidative DNA damage in lung cancer cells: more bad news than good? BMC Cancer. 2018;18(1):423.

25. Lee J, Nho YH, Yun SK, Hwang YS. Anti-invasive and anti-tumor effects of dryopteris crassirhizoma extract by disturbing actin polymerization. Integr Cancer Ther. 2019;18:1534735419851197.

26. Hwang YS, Xianglan Z, Park KK, Chung WY. Functional invadopodia formation through stabilization of the PDPN transcript by IMP-3 and cancer-stromal crosstalk for PDPN expression. Carcinogenesis. 2012;33(11):2135-46. 
27. Glogauer JE, Sun CX, Bradley G, Magalhaes MA. Neutrophils increase oral squamous cell carcinoma invasion through an invadopodia-dependent pathway. Cancer Immunol Res. 2015;3(11):1218-26.

28. Hwang YS, Park KK, Chung WY. Invadopodia formation in oral squamous cell carcinoma: the role of epidermal growth factor receptor signalling. Arch Oral Biol. 2012;57(4):335-43.

29. Qing X, Shao Z, Lv X, et al. Anticancer effect of (S)-crizotinib on osteosarcoma cells by targeting MTH1 and activating reactive oxygen species. Anticancer Drugs. 2018;29(4):341-52.

30. Zhan D, Zhang X, Li J, Ding X, Cui Y, Jia J. MTH1 inhibitor TH287 suppresses gastric cancer development through the regulation of PI3K/AKT signaling. Cancer Biother Radiopharm. 2020;35(3):223-32.

31. Liu X, Fu Y, Huang J, et al. ADAR1 promotes the epithelial-to-mesenchymal transition and stem-like cell phenotype of oral cancer by facilitating oncogenic microRNA maturation. J Exp Clin Cancer Res. 2019;38(1):315.

32. Joseph JP, Harishankar MK, Pillai AA, Devi A. Hypoxia induced EMT: A review on the mechanism of tumor progression and metastasis in OSCC. Oral Oncol. 2018;80:23-32.

33. Dai BW, Yang ZM, Deng P, et al. HOXC10 promotes migration and invasion via the WNT-EMT signaling pathway in oral squamous cell carcinoma. J Cancer. 2019;10(19):4540-51.

\section{Figures}


A

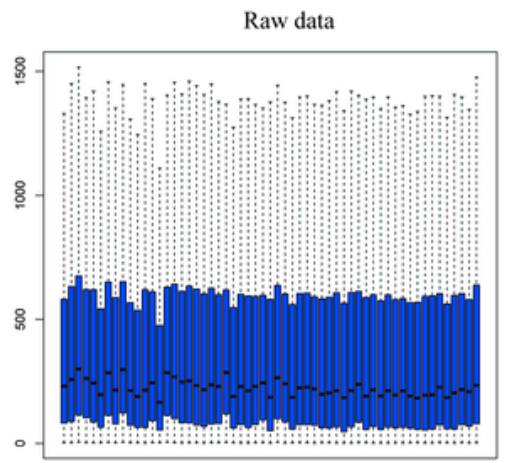

Normalized data

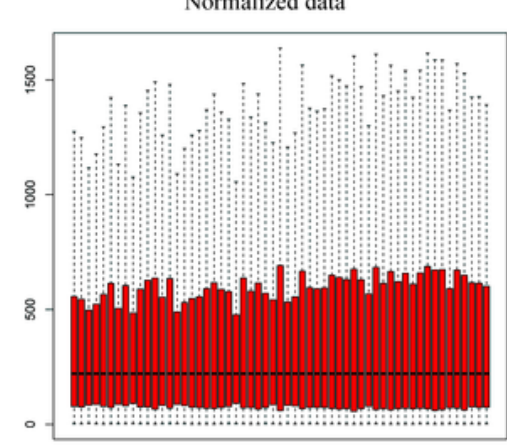

B

GSE13601
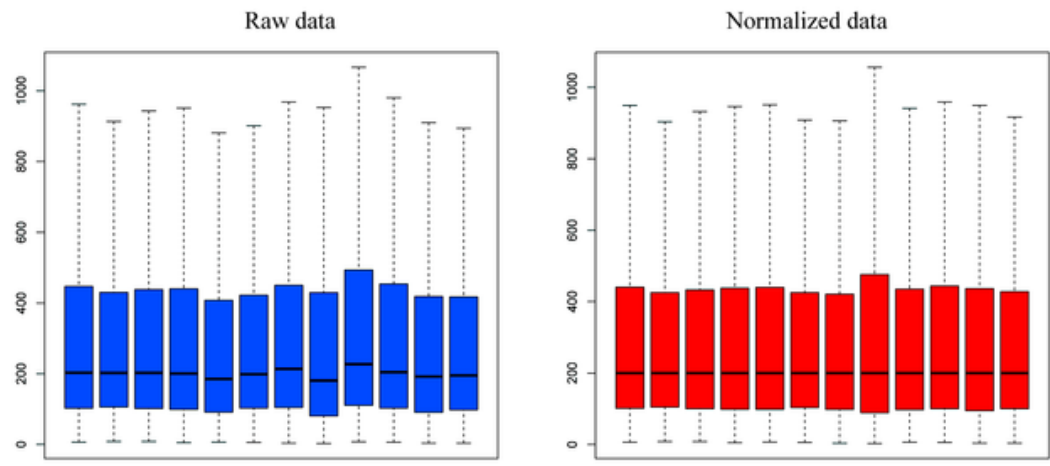

GSE138206

C

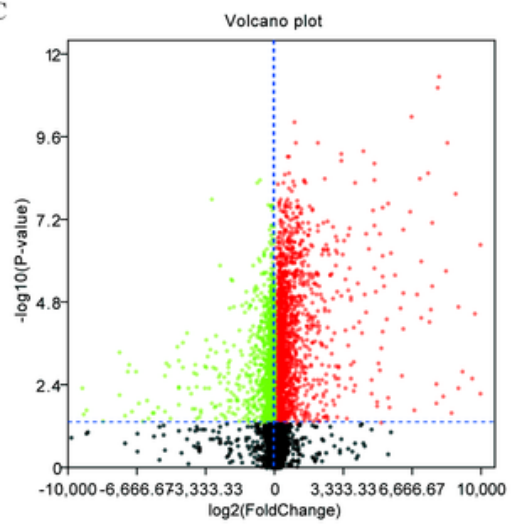

GSE13601
D

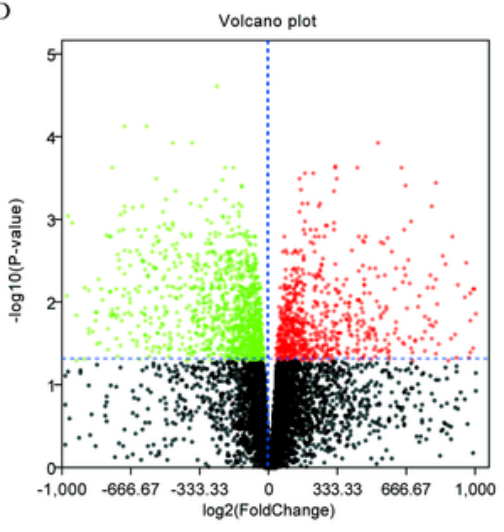

GSE138206

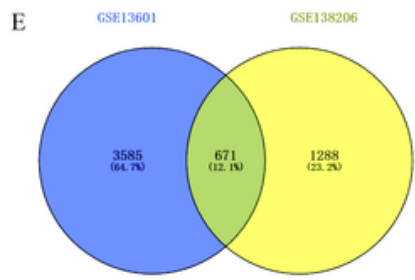

\section{Figure 1}

Identification of common DEGs between GSE13601 and GSE138206. A and B The data of GSE13601 and GSE138206 were normalized by R3.6.2 (limma package). C and D The DEGs in GSE13601 and GSE138206 were presented by Volcano Plots respectively; Green color means down-regulated genes and red color means up-regulated genes. E The common DEGs between GSE13601 and GSE138206 were identified by Venn. 
A

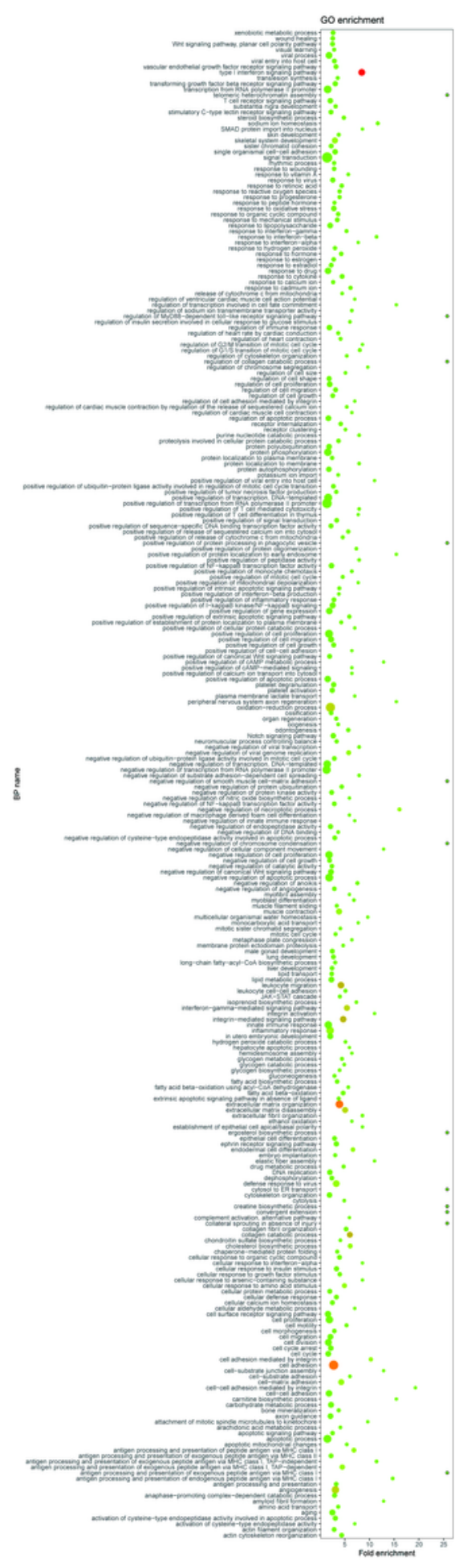

B
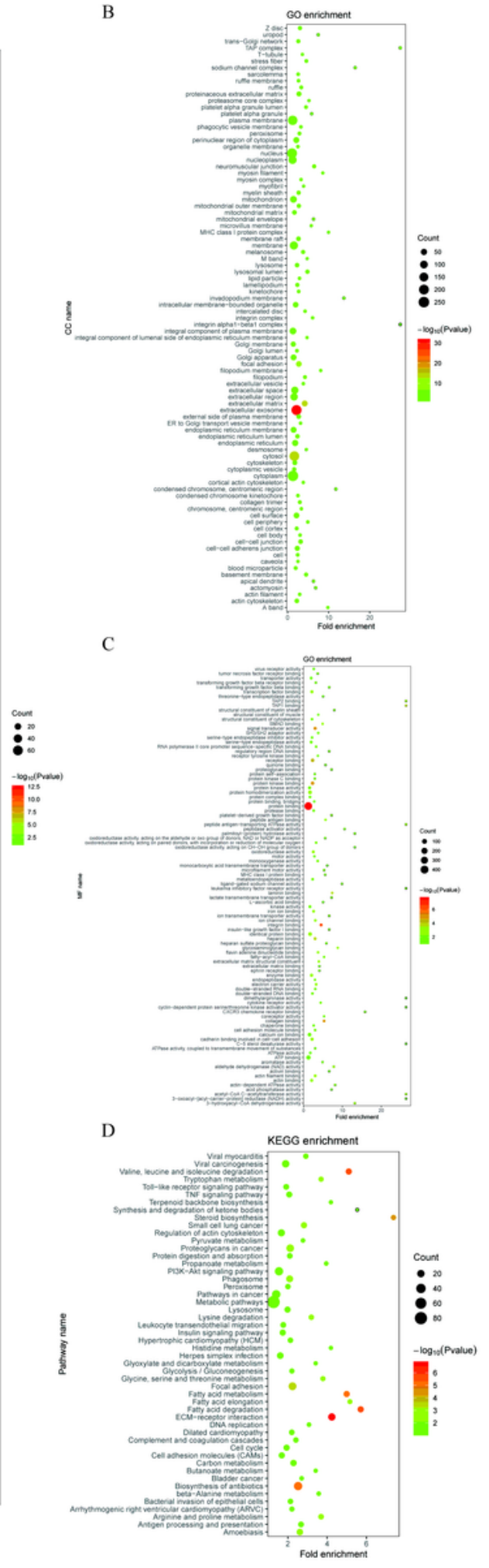

\section{Figure 2}

GO and KEGG enrichment analysis. A, B and C The biological process (A), cell component (B) and molecular function (C) of common DEGs were identified by GO enrichment analysis. $D$ the cell pathways (D) of common DEGs were identified by KEGG enrichment analysis. 
A

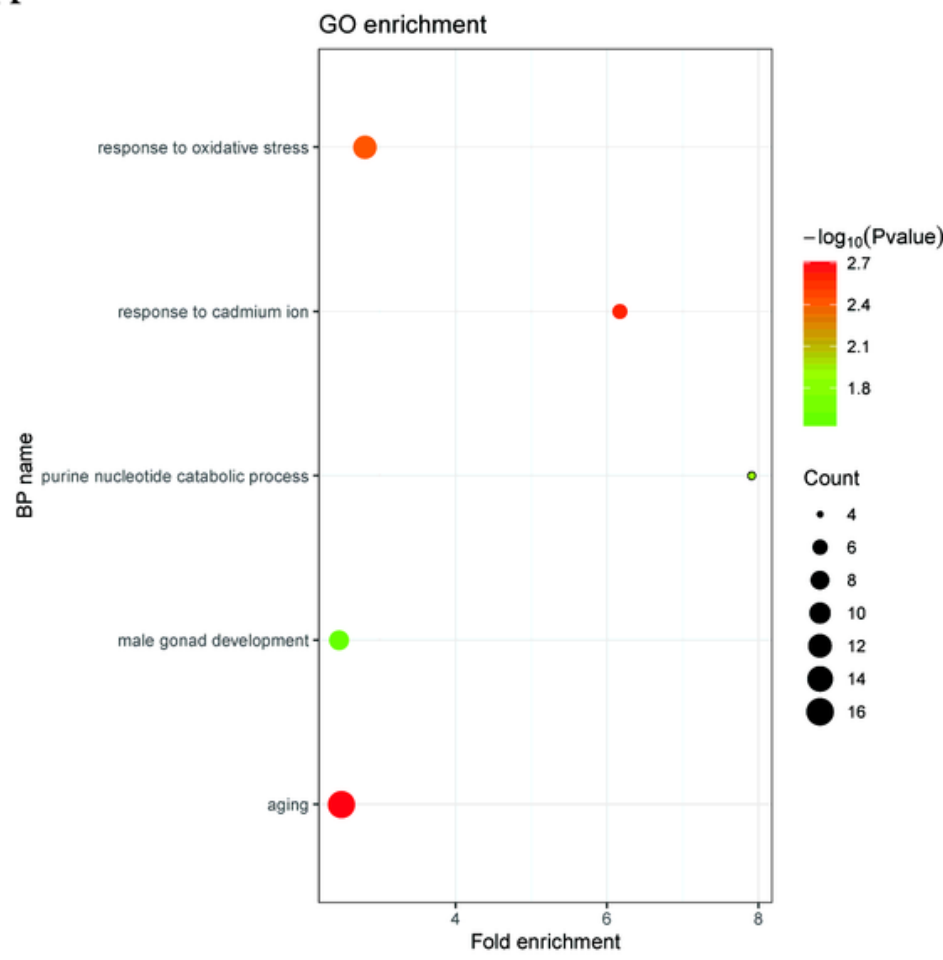

$\mathrm{B}$

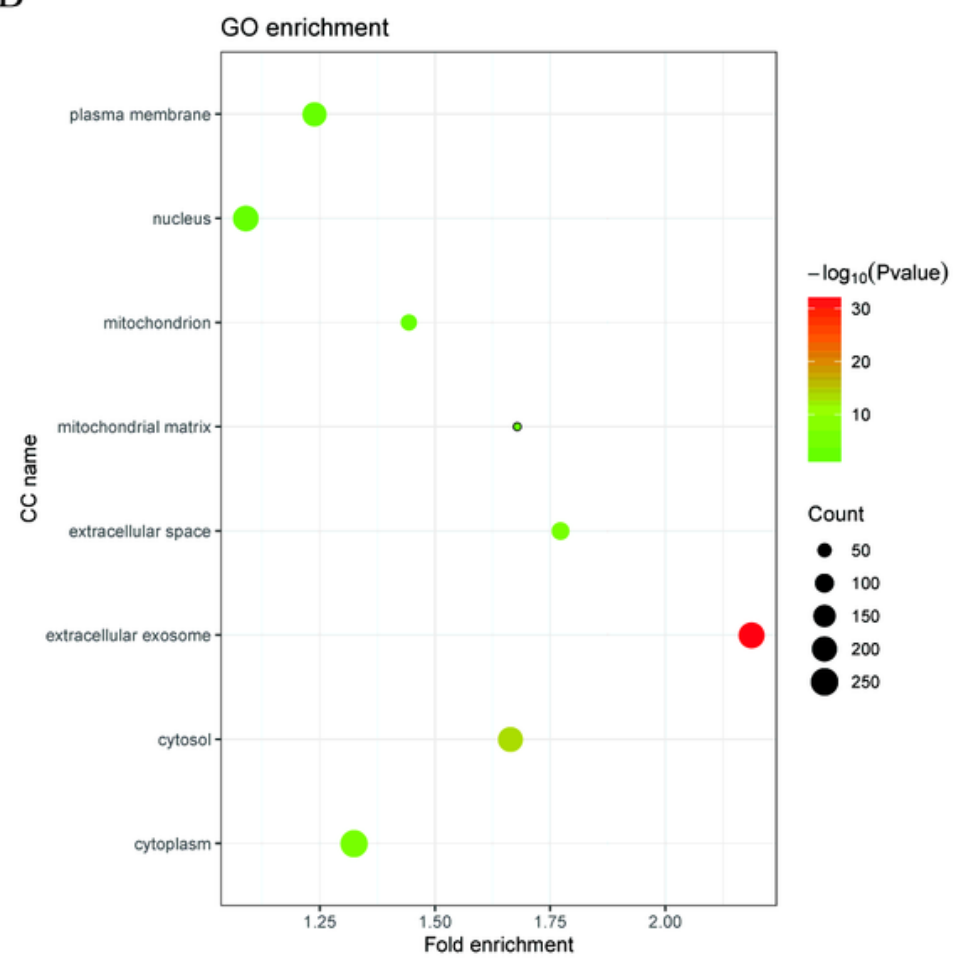

$\mathrm{C}$

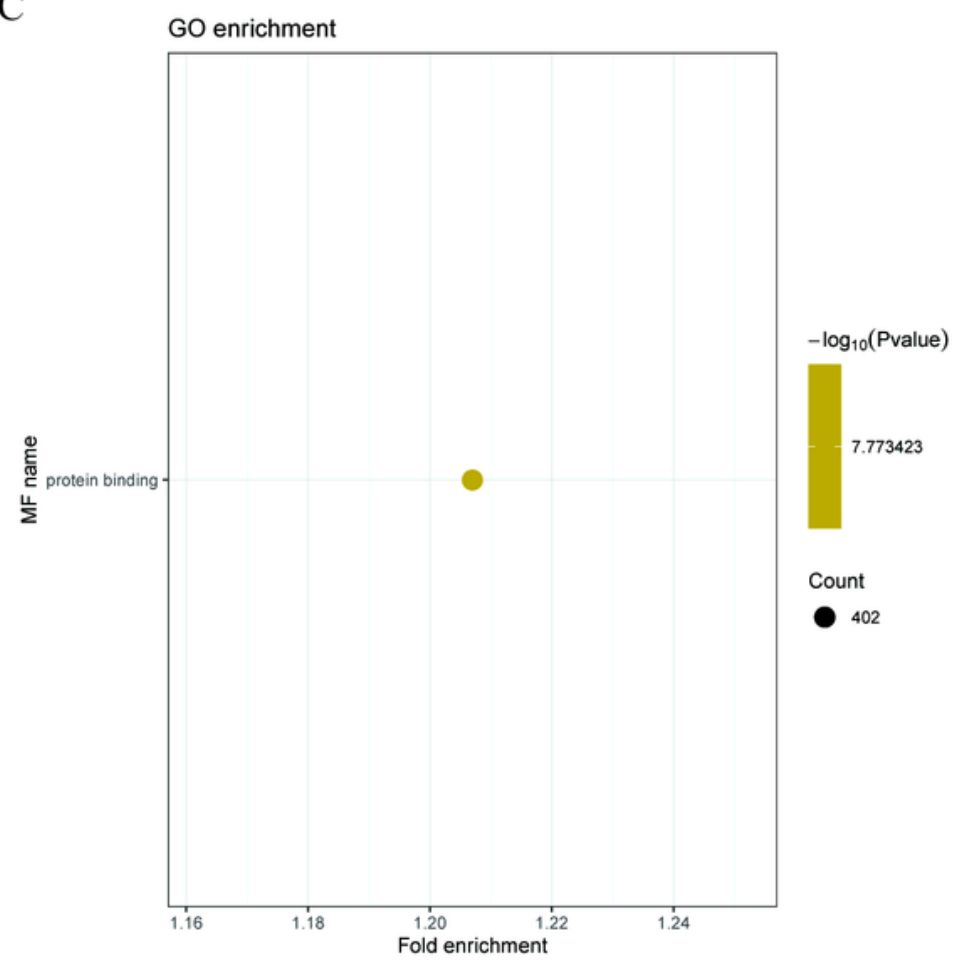

\section{Figure 3}

The biological process, cell component and molecular function of NUDT1 was identified by GO enrichment analysis. A The biological process of NUDT1 was presented by bubble plot. B the cell component of NUDT1 was presented by bubble plot. C the molecular function of NUDT1 was presented by bubble plot. 
A

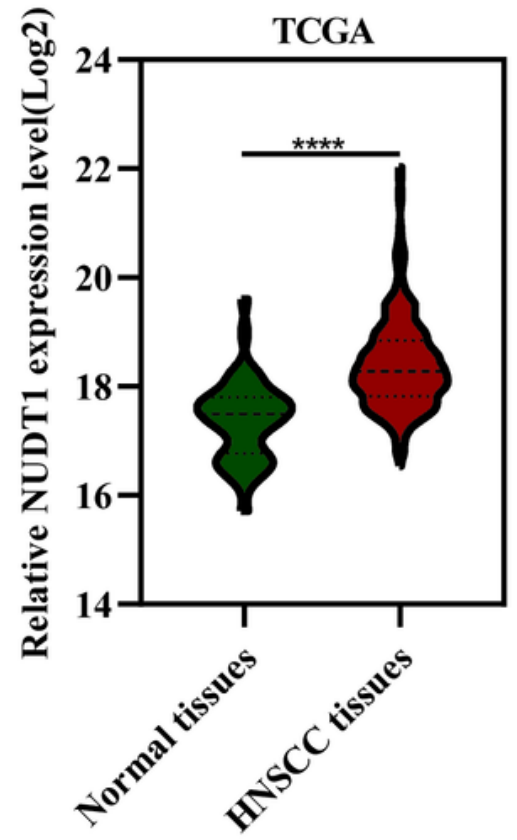

$\mathrm{D}$

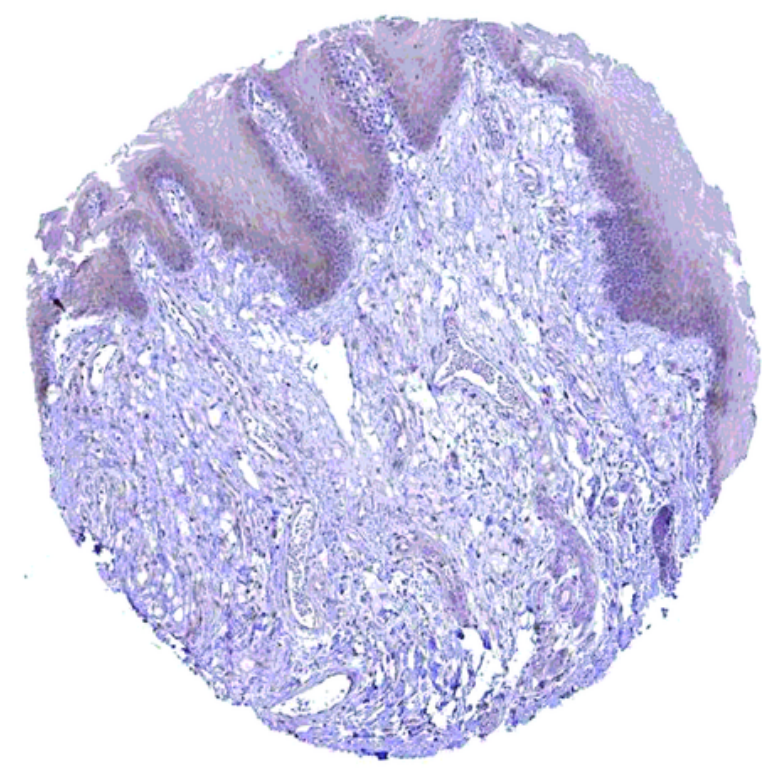

$\mathrm{C}$

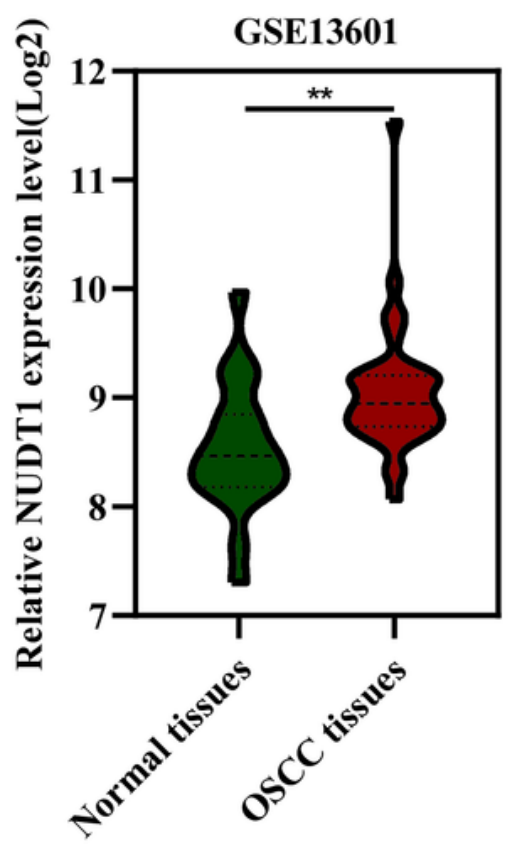

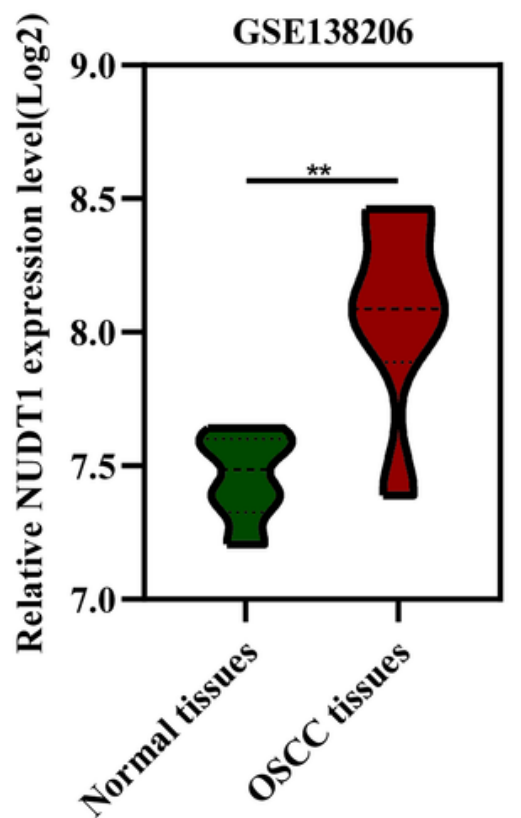

HNSCC tissues

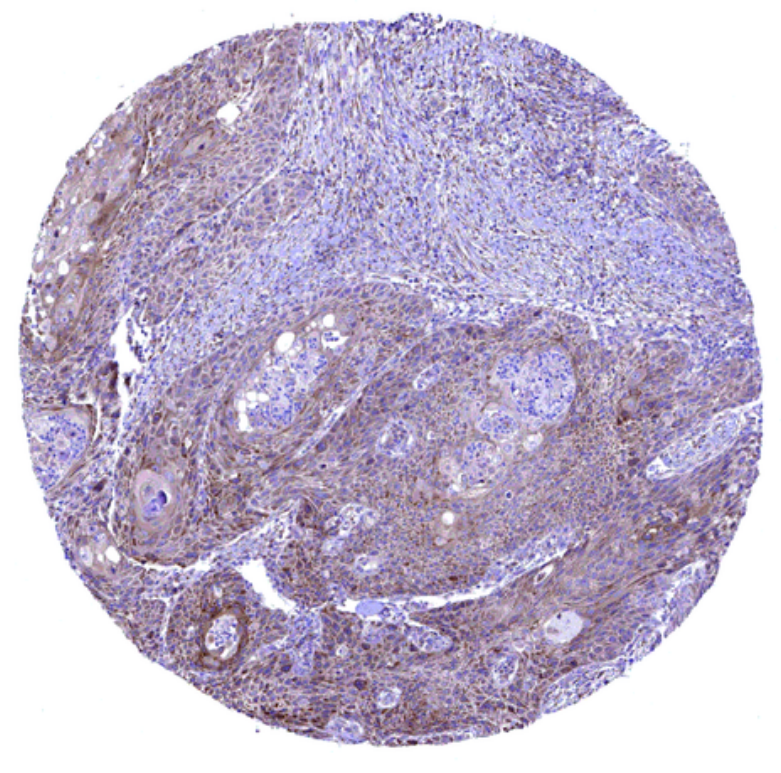

Figure 4

The mRNA and protein expression level of NUDT1 were confirmed by TCGA dataset and The Human Protein Atlas respectively. A, B and C The mRNA expression level of NUDT1 in TCGA dataset, GSE13601 and GSE138206 was presented by violin plots respectively; data are shown as means \pm s.d. (s.d., standard deviation). Statistical analysis was performed by Student's t-test, ** indicates $\mathrm{P}<0.01$, $\star \star \star \star$ indicates $P<0.0001$. D The protein expression level of NUDT1 was confirmed by The Human Protein Atlas. 

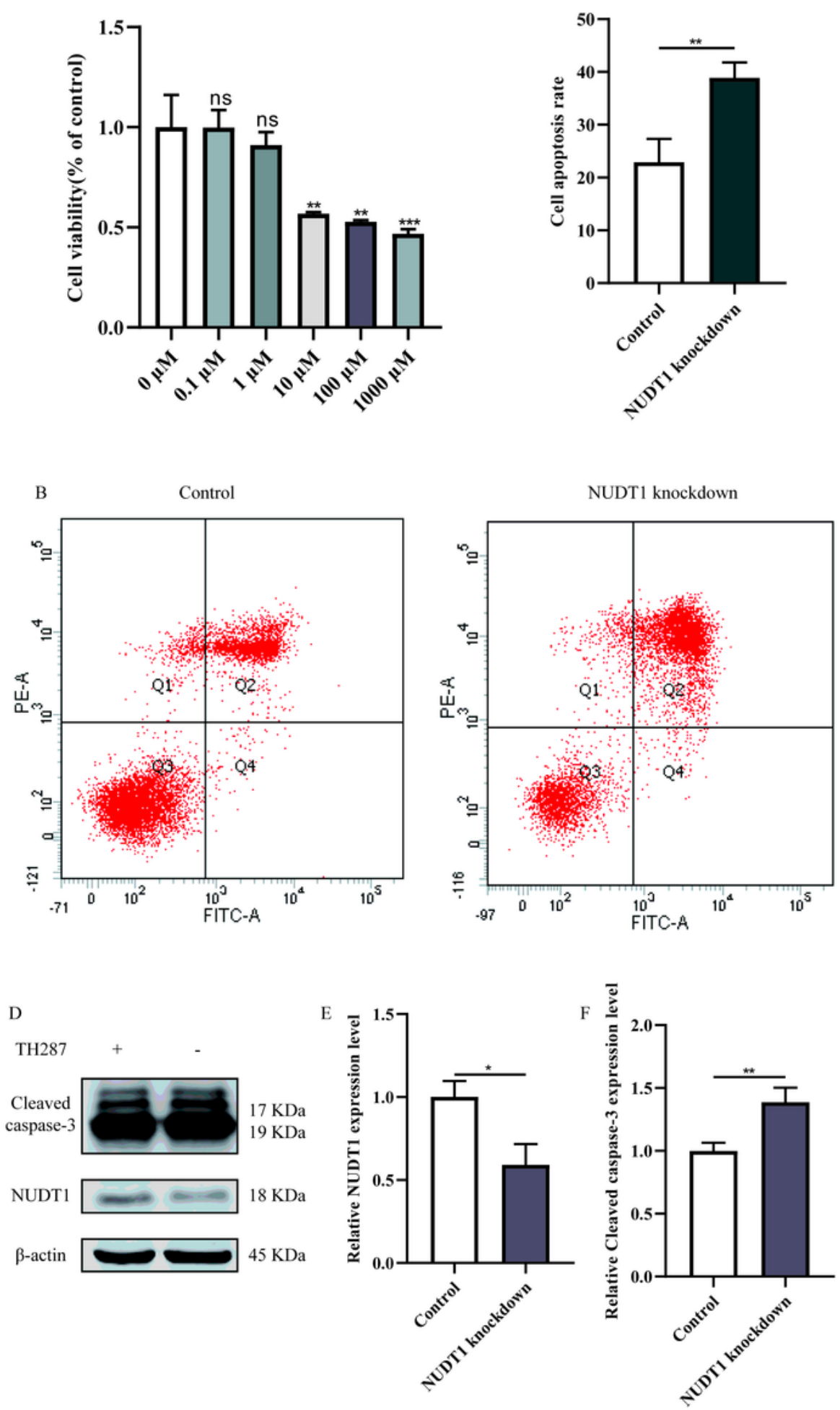

\section{Figure 5}

NUDT1-knockdown inhibited the proliferation of CAL27 by promoting cell apoptosis. A The effect of NUDT1 inhibitor on the proliferation of CAL27 was measured by MTT assay; data are shown as means \pm s.d. (s.d., standard deviation). Statistical analysis was performed by Student's t-test, ns indicates $P>0.05$, ** indicates $P<0.01$, ***indicates $P<0.001$. B The effect of NUDT1 inhibitor on the apoptosis of CAL27 was tested by flow cytometry. C Quantitation of cell apoptosis rate in control group and NUDT1- 
knockdown group; data are shown as means \pm s.d. (s.d., standard deviation). Statistical analysis was performed by Student's t-test, $* *$ indicates $\mathrm{P}<0.01$. D The relative NUDT1 and cleaved caspase-3 protein level were tested by western blot. E and F Quantitation of relative NUDT1 and cleaved caspase-3 protein level in different groups; data are shown as means \pm s.d. (s.d., standard deviation). Statistical analysis was performed by Student's t-test, * indicates $\mathrm{P}<0.05$, ** indicates $\mathrm{P}<0.01$.
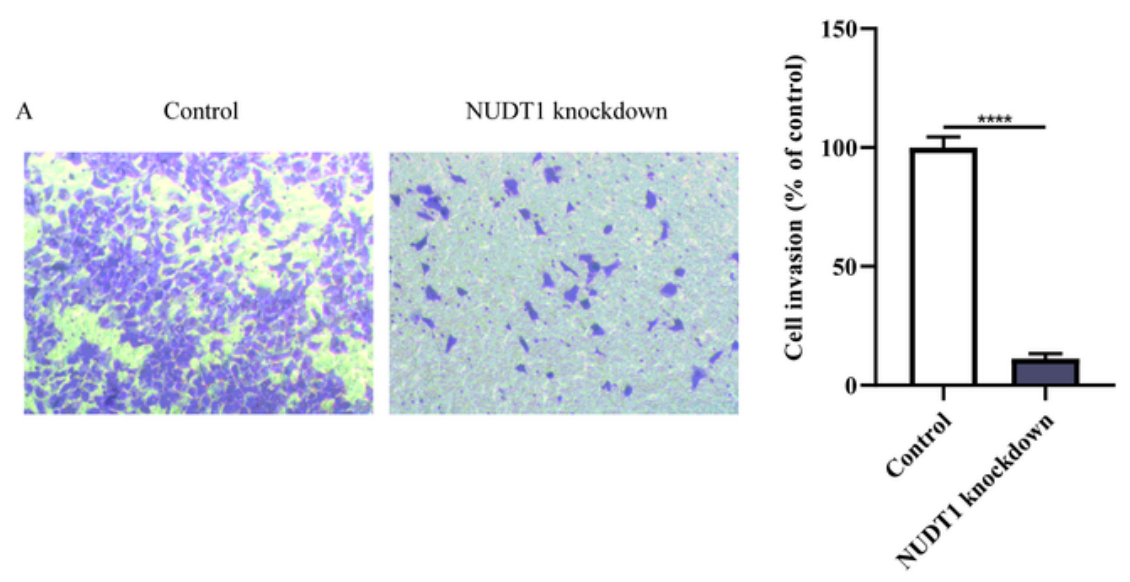

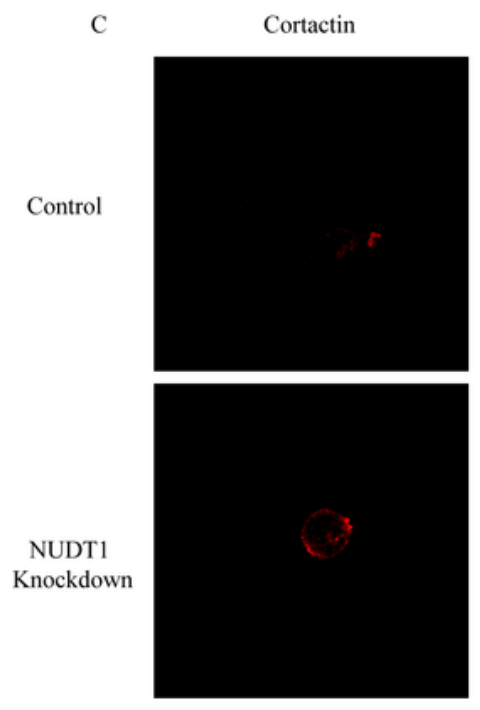

D

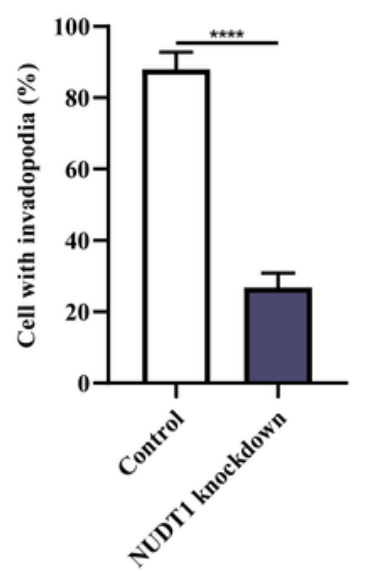

Hoechst
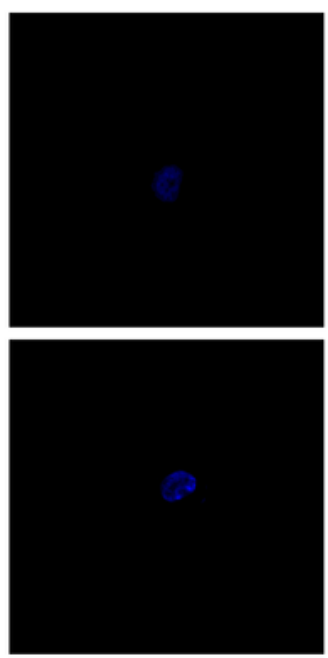

E

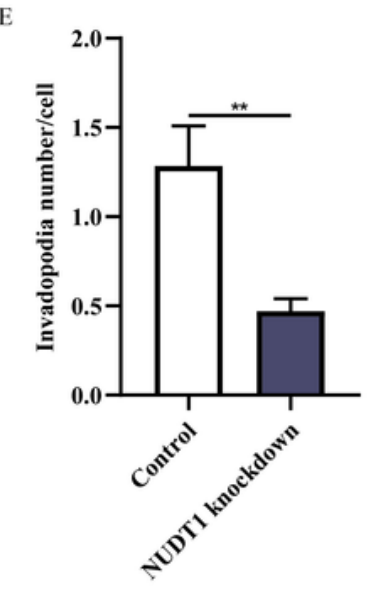

Figure 6 
NUDT1-knockdown inhibited the invasion of CAL27 by suppressing invadopodia formation. A The effect of NUDT1 inhibitor on the invasion of CAL27 was measured by Transwell (Invasion) 200x. B Quantitation of cell invasion rate in control group and NUDT1-knockdown group; data are shown as means \pm s.d. (s.d., standard deviation). Statistical analysis was performed by Student's t-test, $\star \star \star \star$ indicates $P<0.0001$. C The effect of NUDT1 inhibitor on the invadopodia formation was measured by Laser Scanning Microscope 400x. D and E Quantitation of cell with invadopodia and invadopodia number per cell in control group and NUDT1-knockdown group. data are shown as means \pm s.d. (s.d., standard deviation). Statistical analysis was performed by Student's t-test, $* *$ indicates $P<0.01$, $* \star \star \star$ indicates $P<0.0001$. 
A

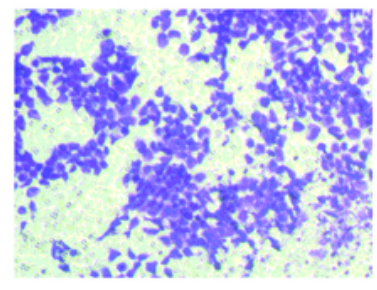

NUDT1 knockdown

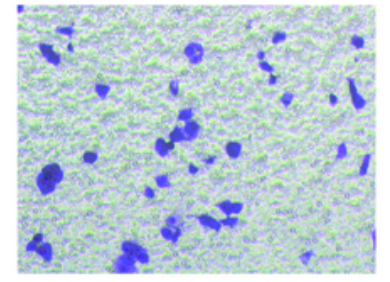

B

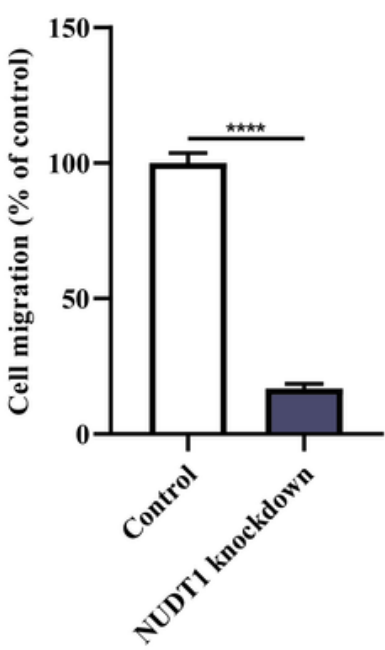

C

$0 \mathrm{~h}$
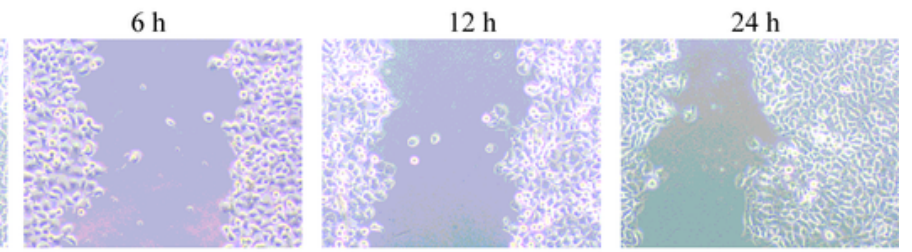

NUDT1

knockdown
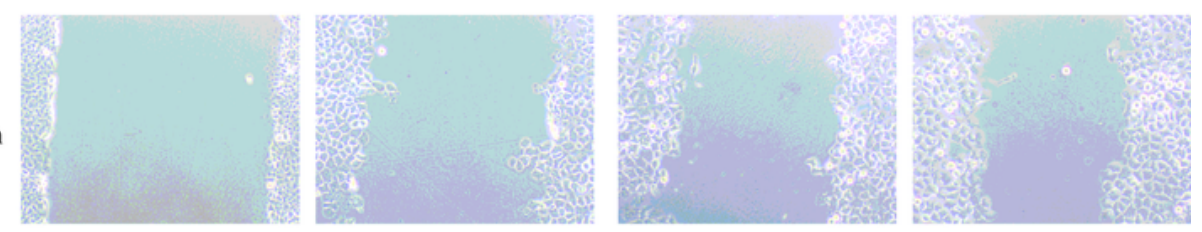

$\mathrm{D}$

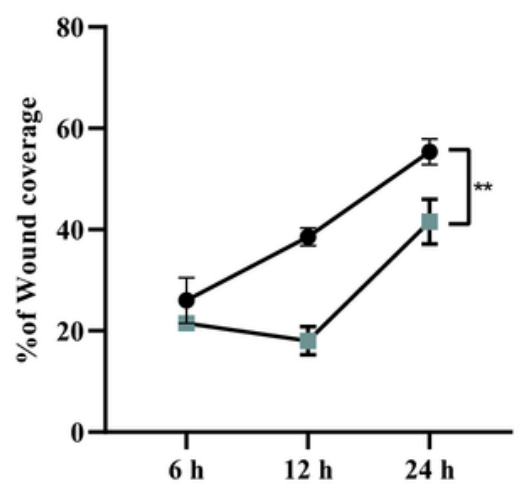

$\rightarrow$ Control

-- NUDT1 knockdown

\section{Figure 7}

NUDT1-knockdown inhibited the migration of CAL27. A and C The effect of NUDT1 inhibitor on the migration of CAL27 was measured by both Transwell(Migration) and Scratch Test 200x. B and D Quantitation of cell migration rate and cell migration distance in control group and NUDT1-knockdown group respectively; data are shown as means \pm s.d. (s.d., standard deviation). Statistical analysis was performed by Student's t-test (B) and Two-way ANOVA (D), ** indicates $P<0.01$, **** indicates $P<0.0001$. 


\section{Supplementary Files}

This is a list of supplementary files associated with this preprint. Click to download.

- Additionalfiles.tif 